\title{
ISL1 promotes cancer progression and inhibits cisplatin sensitivity in triple-negative breast cancer cells
}

\author{
YANG ZHANG $^{1 *}$, LU WANG ${ }^{*}$, PENG GAO $^{2}$, ZHIGUO SUN $^{1}$, NING LI $^{1}$, YANQIN LU $^{1}$, \\ JIANGLUN SHEN $^{1}$, JIAN SUN ${ }^{1}$, YIMING YANG ${ }^{1}$, HAO DAI $^{1}$ and HAIFENG CAI ${ }^{1}$ \\ ${ }^{1}$ The Second Department of Breast Surgery and ${ }^{2}$ The Second Department of Chemoradiotherapy, \\ Tangshan People's Hospital, Tangshan, Hebei 063000, P.R. China
}

Received January 6, 2018; Accepted July 31, 2018

DOI: $10.3892 /$ ijmm.2018.3842

\begin{abstract}
Triple-negative breast cancer (TNBC) is a type of breast cancer that is characterized by the lack of expression of estrogen and progesterone receptors, and epidermal growth factor receptor 2. Therefore, there is an absence of a specific target for effective therapy in TNBC. Cisplatin is usually employed as a first-line chemotherapy agent for patients with TNBC. However, resistance remains an obstacle for cisplatin-based chemotherapy, due to its elusive underlying mechanism. Previously, abnormal expression of Islet 1 (ISL1) was demonstrated to be closely associated with cancer development and progression. The present study revealed that (ISL1) was significantly upregulated in TNBC tissues in comparison with adjacent normal tissues. Overexpression of ISL1 markedly promoted the proliferation and invasion of the TNBC MDA-MB-231 and MDA-MB-468 cell lines, while knockdown of ISL1 inhibited cell invasion and proliferation in these cell lines. In addition, overexpression of ISL1 reversed cisplatin-induced cell apoptosis, while knockdown of ISL1 enhanced apoptosis following cisplatin treatment in MDA-MB-231 and MDA-MB-468 cells. Furthermore, the levels of the anti-apoptotic proteins, phosphorylated-protein kinase B and B-cell lymphoma-2 (Bcl-2), were significantly decreased, while the levels of the pro-apoptotic protein Bcl-2-associated $\mathrm{X}$ protein were remarkably increased in response to cisplatin treatment. The present study revealed that ISL1 overexpression reversed the protein expression profile of p-Akt, Bcl-2 and Bax, while ISL1 knockdown promoted cell apoptosis. Therefore, the data of the present study demonstrated that ISL1 contributes to TNBC progres-
\end{abstract}

Correspondence to: Dr Haifeng Cai, The Second Department of Breast Surgery, Tangshan People's Hospital, 65 Shengli Road, Lunan, Tangshan, Hebei 063000, P.R. China

E-mail: caihaifengts@aliyun.com

*Contributed equally

Key words: triple-negative breast cancer, cisplatin sensitivity, islet 1 , cancer progression sion and reverses cell sensitivity towards cisplatin in TNBC cells, suggesting that ISL1 is a potential therapeutic target for the treatment of TNBC.

\section{Introduction}

Breast cancer remains the leading cause of mortality in females worldwide (1). Based on the expression of estrogen and progesterone receptors, and epidermal growth factor receptor 2 (Her2), breast cancer is classified into 4 sub-types as follows: Luminal A, luminal B, Her2-overexpressing and triple-negative breast cancer (TNBC) (2). TNBC comprises a heterogeneous group of cancer types (3). With no effective therapeutic target, the current treatment of TNBC relies on combination therapy, including chemotherapy (4). Although TNBC usually responds well to chemotherapy, de novo and acquired chemotherapy resistance remain to be overcome in order to achieve an improved overall survival for patients with TNBC (5). Notably, tumor metastasis is an additional frequent obstacle when treating TNBC $(6,7)$.

Cisplatin is a commonly used chemotherapeutic agent administered to patients with TNBC (8). The antitumor properties of cisplatin are primarily based on its ability to induce cell apoptosis by causing DNA damage (9). However, the efficacy of cisplatin is frequently compromised by the insensitivity of malignant cells towards drug treatment and the development of drug resistance $(10,11)$. The underlying mechanism of cisplatin resistance is complex. Previous studies on cancer cell lines indicated that the activity of the p38 mitogen-activated protein kinase signaling pathway was associated with cisplatin sensitivity (12). An additional study revealed that protein kinase B (Akt) was involved in cisplatin-resistance by inhibiting cell apoptosis (13). Consequently, future studies on the precise molecular mechanisms of cisplatin sensitivity are required to meet current clinical requirements.

Islet 1 (ISL1) is a member of the LIM/homeodomain family of transcription factors and was first cloned from pancreatic insulin-producing cells of rats $(14,15)$. Through binding the insulin gene enhancer, ISL1 was identified to regulate insulin gene expression (14). ISL1 is involved in the development of numerous tissue types, including the nervous system, pancreas and skeletal muscles (15). Previously, abnormal expression of ISL1 has been demonstrated to be 
closely associated with cancer development and progression (16). Immunohistochemical staining of breast cancer samples revealed that the protein levels of ISL1 were increased in tumor tissues from patients with TNBC compared with those in other breast cancer sub-types (17). However, the role of ISL1 in TNBC progression, and its underlying mechanism, remains unknown.

The present study aimed to explore the role of ISL1 in TNBC. The results of reverse transcription-quantitative polymerase chain reaction (RT-qPCR) analysis revealed that ISL1 expression was significantly increased in TNBC tissues compared with that in normal adjacent tissues. The present study also demonstrated that ISL1 markedly promoted cell proliferation and invasion in the TNBC MDA-MB-231 and MDA-MB-468 cell lines. Additionally, overexpression of ILS1 markedly reversed cisplatin-induced cell apoptosis in MDA-MB-231 and MDA-MB-468 cells. Furthermore, ILS1 inhibited cell apoptosis via upregulation of the expression of the anti-apoptotic proteins, phosphorylated-Akt (p-Akt) and B-cell lymphoma-2 (Bcl-2), and downregulation of the expression of the pro-apoptotic protein, Bcl-2-associated $\mathrm{X}$ protein (Bax). Taken together, these data suggested that dysregulation of ILS1 participates in TNBC cell progression and sensitivity to cisplatin, proposing ILS1 as a promising therapeutic target in TNBC.

\section{Materials and methods}

Patients. Tumor tissues and their corresponding adjacent $(>5 \mathrm{~cm}$ ) normal tissues were obtained from 35 patients with TNBC who attended Tangshan People's Hospital (Tangshan, China) from March, 2012 to September, 2015. In the present cohort, there were 9 patients $<35$ years old and 26 patients $>35$ years old (28 years old-65 years old). All tissues were stored at $-80^{\circ} \mathrm{C}$ prior to the extraction of nucleic acids. Written informed consent for use of patient samples was obtained from all participants in the present study prior to surgery. The experiments were performed following approval from the Ethics Committee of Tangshan People's Hospital.

Cell culture and reagents. The human TNBC MDA-MB-231 and MDA-MB-468 cell lines, and 293 cell line were purchased from American Type Culture Collection (Manassas, VA, USA). The 293, MDA-MB-231 and MDA-MB-468 cells were cultured in Dulbecco's modified Eagle's medium (DMEM; Thermo Fisher Scientific, Inc., Waltham, MA, USA) supplemented with $10 \%$ fetal bovine serum (FBS; HyClone; GE Healthcare Life Sciences, Logan, UT, USA), 100 U/ml penicillin and $100 \mu \mathrm{g} / \mathrm{ml}$ streptomycin (Thermo Fisher Scientific, Inc.) in an incubator with $5 \% \mathrm{CO}_{2}$ at $37^{\circ} \mathrm{C}$.

Cisplatin was purchased from Sigma-Aldrich (Merck KGaA, Darmstadt, Germany). For cisplatin treatment, MDA-MB-231 and MDA-MB-468 cells ( $3 \times 10^{3}$ cells/well) were seeded into 96-well plates (Sarstedt, Nümbrecht, Germany) and incubated with $25 \mu \mathrm{M}$ cisplatin in DMEM in an incubator with $5 \% \mathrm{CO}_{2}$ at $37^{\circ} \mathrm{C}$ for $24 \mathrm{~h}$, and then subjected to subsequent analyses.

ISL1 overexpression. The full-length sequence of ISL1 was amplified from cDNA of 293 cells and cloned into the
pcDNA3 plasmid (Addgene, Inc., Cambridge, MA, USA). For ISL1 overexpression, $1 \times 10^{6}$ MDA-MB-231 and MDA-MB-468 cells were transfected with $2 \mu \mathrm{g}$ pcDNA3-ISL1 using Lipofectamine ${ }^{\circledR} 2000$ (Thermo Fisher Scientific, Inc.), while cells transfected with empty vector served as controls. After $24 \mathrm{~h}$, the cells were subjected to following experiments.

RNA interference. MDA-MB-231 and MDA-MB-468 cells were transfected with a scramble small interfering RNA (siRNA; $100 \mathrm{nM}$ ) used as negative control or with ISL1 siRNA pool (containing three specific siRNAs) (100 nM; cat. no. sc-37121, Santa Cruz Biotechnology, Inc., Dallas, TX, USA) using Lipofectamine RNAi MAX (Thermo Fisher Scientific, Inc.), and incubated at $37^{\circ} \mathrm{C}$ for $72 \mathrm{~h}$. Following this, cells were subjected to subsequent experiments. Sequences of siRNAs were listed as follow: Control siRNA: 5'-UUCUCC GAACGUGUCACGU-3'; ISL1 siRNA pool: 5'-GGACCA GGCUCUAAUUCUATT-3'; 5'-AAAAGAAUGGAGGUG GAAG; GAGACAUGGUGGUUUA-3'.

Cell proliferation assay. Cell proliferation was assessed with a Cell-Counting-Kit-8 (CCK-8; Dojindo Molecular Technologies, Inc., Kumamoto, Japan), according to the manufacturer's protocols. Briefly, MDA-MB-231 and MDA-MB-468 cells $\left(3 \times 10^{3}\right.$ cells/well) were seeded into 96 -well plates. Following cell attachment, cells were treated $\left(100 \mathrm{nM}, 37^{\circ} \mathrm{C}\right)$ with control siRNA, ISL1 siRNA, pcDNA3 or pcDNA3-ISL1 for $72 \mathrm{~h}$. To evaluate the effect of ISL1 in cell proliferation, cells were treated $\left(37^{\circ} \mathrm{C}\right)$ with vehicle (dimethyl sulfoxide; Beijing Solarbio Science \& Technology, Co., Ltd., Beijing, China), $10 \mu \mathrm{M}$ cisplatin, $10 \mu \mathrm{M}$ cisplatin + pcDNA3-ISL1 or $10 \mu \mathrm{M}$ cisplatin + ISL1 siRNA for $72 \mathrm{~h}$. Next, CCK-8 solution $(10 \mu \mathrm{l})$ was added into each well and incubated $\left(37^{\circ} \mathrm{C}\right)$ for $2 \mathrm{~h}$. Subsequently, the absorbance at $450 \mathrm{~nm}$ was recorded to determine cell proliferation.

Cell invasion assay. Cell invasion ability was measured by a Transwell assay. Briefly, $3 \times 10^{4}$ MDA-MB-231 and MDA-MB-468 cells were plated onto the upper side of Matrigel-coated $\left(30 \mathrm{~min}\right.$ at $\left.37^{\circ} \mathrm{C}\right)$ Transwell chambers (Corning Incorporated, Corning, NY, USA) with serum-free DMEM, while the lower chamber was filled with DMEM containing $10 \%$ FBS. Following culture $\left(37^{\circ} \mathrm{C}\right)$ for $24 \mathrm{~h}$, the cells in the upper chamber were removed by a cotton swab, while the cells that had invaded into the reverse face of the membranes were fixed ( $1 \mathrm{~h}$ at room temperature) with methanol (4\%) and stained ( $1 \mathrm{~h}$ at room temperature) with crystal violet $(0.25 \%)$. Images were captured from 3 randomly chosen fields and the number of cells was then quantified using a light microscope (SZX16-3111; Olympus Corporation, Tokyo, Japan). The experiment was repeated three times.

Cell apoptosis assay. Cell apoptosis was detected with the Fluorescein isothiocyanate-Annexin V/Apoptosis Detection kit (Thermo Fisher Scientific, Inc.) with a BD FACSCalibur flow cytometer (BD Biosciences, Franklin Lakes, NJ, USA). Upon treatment of control siRNA or ISL1 siRNA, and vehicle, $10 \mu \mathrm{M}$ cisplatin, $10 \mu \mathrm{M}$ cisplatin + pcDNA3-ISL1 or $10 \mu \mathrm{M}$ cisplatin + ISL1 siRNA, the MDA-MB-231 and MDA-MB-468 cells were harvested, re-suspended in $500 \mu \mathrm{l}$ pre-cooled $1 \mathrm{X}$ binding 
buffer from Fluorescein isothiocyanate-Annexin V/Apoptosis Detection kit, and mixed with $5 \mu \mathrm{l}$ Annexin V and $2.5 \mu \mathrm{l}$ propidium iodide (PI). The scatter plots contained the following results: Quadrant 4 (Q4), healthy cells (Annexin $\left.\mathrm{V}^{-} / \mathrm{PI}^{-}\right)$; Q3, apoptotic cells at an early stage (Annexin $\left.\mathrm{V}^{+} / \mathrm{PI}^{-}\right)$; $\mathrm{Q} 2$, apoptotic cells at an advanced stage (Annexin $\left.\mathrm{V}^{+} / \mathrm{PI}^{+}\right)$; and $\mathrm{Q} 1$, mechanically injured cells (Annexin $\mathrm{V}^{-} / \mathrm{PI}^{+}$). Flow cytometry results were analyzed using FlowJo 10.4.2 (FlowJo LLC, Ashland, OR, USA). The apoptotic rate was calculated as the ratio of apoptotic cells in Q3 + Q2 to the total number of cells.

Western blotting. Antibodies against ISL1 (cat. no. sc-390793; 1:1,000), Bcl-2 (cat. no. sc-7382; 1:1,000) and Bax (cat. no. sc-7480; 1:1,000) were purchased from Santa Cruz Biotechnology, Inc. The antibody against GAPDH was obtained from Sigma-Aldrich (Merck KGaA). Secondary antibodies against mouse (cat. no. SA00001-1; 1:10,000) and rabbit (cat. no. SA00001-1; 1:10,000) were products of Proteintech Group,Inc.,(Chicago,IL, USA). Western blotting was performed following standard procedures: In brief, protein lysates from MDA-MB-231 cells were prepared using radioimmunoprecipitation assay lysis buffer (Beyotime Institute of Biotechnology, Haimen, China), quantified by the method of BCA (Beyotime Institute of Biotechnology, Shanghai, China), $20 \mu \mathrm{g}$ protein per line were separated by $8 \%$ SDS-PAGE and then transferred to polyvinylidene fluoride membranes. Subsequently, the membranes were blocked ( $1 \mathrm{~h}$ at room temperature) with $5 \%$ non-fat milk (5 g milk powder in $100 \mathrm{ml}$ TBST with $0.1 \%$ Tween 20) and incubated ( $1 \mathrm{~h}$ at room temperature) with the aforementioned primary and secondary antibodies. The blots were developed with SuperSignal West Femto Maximum Sensitivity Substrate (Thermo Fisher Scientific, Inc.). GAPDH served as an internal control. Quantitative analysis of protein expression was achieved by Image J version 1.8.0 (National Institutes of Health, Bethesda, MD, USA).

$R T-q P C R$. Total RNA was extracted from patient tissues with TRIzol $^{\circledR}$ reagent (Life Technologies; Thermo Fisher Scientific, Inc.). The concentration and quality of the extracted RNA was analyzed by NanoDrop 2000 (Thermo Fisher Scientific, Inc., Wilmington, DE, USA). The RNA was reverse transcribed into cDNA using the PrimeScript RT Reagent kit (Takara Bio, Inc., Otsu, Japan). RT-qPCR was performed using the SYBR Green kit (Applied Biosystems; Thermo Fisher Scientific, Inc.) and an ABI Prism 7500 Sequence Detection system (Applied Biosystems; Thermo Fisher Scientific, Inc.). GAPDH served as an internal control. Thermocycling conditions used in the RT-qPCR was as follows: Denaturation: $95^{\circ} \mathrm{C}$ for $10 \mathrm{~min}$, followed by annealing and elongation: 40 cycles at $95^{\circ} \mathrm{C}$ for $15 \mathrm{sec}, 60^{\circ} \mathrm{C}$ for $60 \mathrm{sec}$, and final elongation: 1 cycle at $95^{\circ} \mathrm{C}$ for $15 \mathrm{sec}$. The qPCR primer sequences were as follows: ISL1-forward, 5'-CTGCTTTTCAGCAACTGG TCA-3'; ISL1-reverse, 5'-TAGGACTGGCTACCATGCTGT-3'; GAPDH-forward, 5'-GGAGCGAGATCCCTCCAAAAT-3'; and GAPDH-reverse, 5'-GGCTGTTGTCATACTTCTCA TGG-3'. The relative expression of gene was analyzed using the $2^{-\Delta \Delta \mathrm{Cq}}$ method (18).

Immunohistochemistry. Tumor tissues and the adjacent normal tissues from patients were first sliced into $4 \mu \mathrm{m}$-thick sections.
Afterwards, sections were fixed $\left(24 \mathrm{~h}\right.$ at $\left.4^{\circ} \mathrm{C}\right)$ using $7.5 \%$ buffered formalin and embedded with paraffin. Then deparaffinization and antigen retrieval [in $(\mathrm{pH}$ 6.0) citrate buffer at $98^{\circ} \mathrm{C}$ for $5 \mathrm{~min}$, in $3 \% \mathrm{H}_{2} \mathrm{O}_{2}$ for $15 \mathrm{~min}$ at $98^{\circ} \mathrm{C}$ in a dark place] were performed prior to incubation with the primary ISL1 antibody (cat. no. sc-390793; 1:50) overnight at $4^{\circ} \mathrm{C}$. Subsequently, slides were incubated with horseradish peroxidase-conjugated secondary antibody (ZSGB-BIO; OriGene Technologies, Inc., Beijing, China) at $37^{\circ} \mathrm{C}$ for $30 \mathrm{~min}$. The 3,3-diaminobenzidine detection kit (Wuhan Boster Biological Technology, Ltd., Wuhan, China) acted as the final chromogen and the nucleus was counterstained (3 $\mathrm{min}$ at room temperature) with hematoxylin $(0.5 \%)$. Images were captured from at least 6 representative fields of view at magnification, $x 400$. The immunostaining was assessed by two independent pathologists from Tangshan People's Hospital with no knowledge of the groups.

Statistical analysis. All data were analyzed using GraphPad Prism 6.0 (GraphPad Software, Inc., La Jolla, CA, USA) and expressed as the mean \pm standard deviation. Comparisons between two groups and multiple groups were performed by unpaired Student's t-test and one-way analysis of variance followed by a Student-Newman-Keuls test, respectively. The $\chi^{2}$ test was applied for the analysis of the association between ISL1 and clinicopathological characteristics of patients. $\mathrm{P}<0.05$ was considered to indicate a statistically significant difference. Each experiment was replicated three times.

\section{Results}

ISL1 is overexpressed in tumor tissues of patients with TNBC. To identify whether ISL1 was increased in tumor tissues, the present study detected mRNA expression levels of ISL1 in tumor tissues and normal adjacent tissues in 35 patients with TNBC by RT-qPCR. As indicated in Fig. 1, ISL1 mRNA and protein levels were significantly increased in tumor tissues compared with those in normal adjacent tissues.

In addition, the association between changes in ISL1 expression levels and the clinicopathological characteristics of the patients are summarized in Table I. There was no significant difference in ISL1 levels between $<35$-year-old patients $(3.15 \pm 1.35)$ and those aged $\geq 35$-years $(3.37 \pm 1.21 ; \mathrm{P}=0.64)$. There were 17 patients whose tumor-size was $<4 \mathrm{~cm}$ and 18 patients whose tumor-size was $\geq 4 \mathrm{~cm}$, but no significant difference in ISL1 levels was observed between these two groups $(3.39 \pm 1.25$ vs. $3.24 \pm 1.97$, respectively; $\mathrm{P}=0.71)$. According to their histological grade by the Nottingham modification of the Scarff-Bloom-Richardson (NSBR) grading scheme (19), patients were grouped into well/intermediate differentiation $(n=14)$ and poor differentiation groups $(n=21)$, but no significant difference in ISL1 expression levels was observed between the two groups $(3.45 \pm 1.11$ vs. $3.22 \pm 1.67$, respectively; $\mathrm{P}=0.60)$. In total, 22 patients exhibited Tis-T2 invasion depth, while 13 patients exhibited T3-T4 invasion depth (19). The expression levels of ISL1 in these patients were 3.17 \pm 1.37 and $3.56 \pm 1.77$, respectively, with no significant difference between the two groups $(\mathrm{P}=0.36)$. By contrast, a significant difference in ISL1 levels was observed for lymph node metastasis between N0 and N1-N3 (2.71 \pm 0.85 vs. $3.59 \pm 1.24 ; \mathrm{P}=0.04)$. Similarly, a 
Table I. Association between ISL1 expression and clinicopathological parameters of patients with breast cancer.

\begin{tabular}{|c|c|c|c|}
\hline Clinicopathological parameters & $\mathrm{N}$ & ISL1 (fold-change) & $\mathrm{P}$-value \\
\hline Age, years & & & 0.64 \\
\hline$<35$ & 9 & $3.15 \pm 1.35$ & \\
\hline$\geq 35$ & 26 & $3.37 \pm 1.21$ & \\
\hline Tumor size, $\mathrm{cm}$ & & & 0.71 \\
\hline$<4$ & 17 & $3.39 \pm 1.25$ & \\
\hline$\geq 4$ & 18 & $3.24 \pm 1.97$ & \\
\hline Histological grade & & & 0.60 \\
\hline Well/intermediate differentiation & 14 & $3.45 \pm 1.11$ & \\
\hline Poor differentiation & 21 & $3.22 \pm 1.67$ & \\
\hline Invasion depth & & & 0.36 \\
\hline Tis-T2 & 22 & $3.17 \pm 1.37$ & \\
\hline T3-T4 & 13 & $3.56 \pm 1.77$ & \\
\hline Lymph node metastasis & & & $0.04^{\mathrm{a}}$ \\
\hline N0 & 11 & $2.71 \pm 0.85$ & \\
\hline N1-N3 & 24 & $3.59 \pm 1.24$ & \\
\hline Distant metastasis & & & $0.04^{\mathrm{a}}$ \\
\hline M0 & 29 & $3.13 \pm 1.25$ & \\
\hline M1 & 6 & $4.22 \pm 1.49$ & \\
\hline
\end{tabular}

${ }^{\mathrm{a}}<0.05$. Tis, tumor in situ. ISL1, Islet 1.
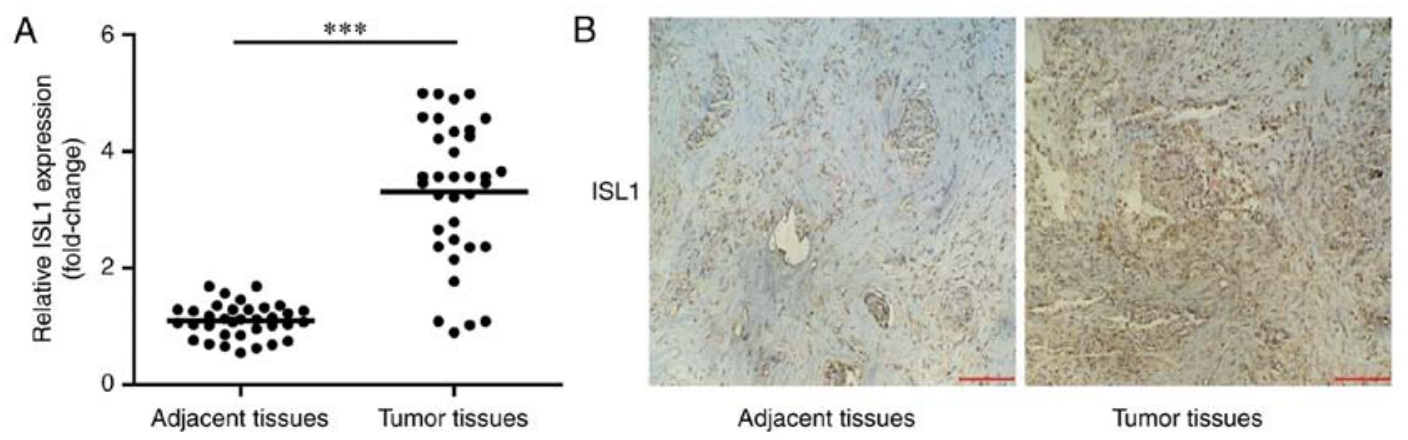

Figure 1. ISL1 is overexpressed in tumor tissues from patients with triple-negative breast cancer. (A) The mRNA levels of ISL1 in tumor tissues and normal adjacent tissues were detected using reverse transcription quantitative polymerase chain reaction. Significant increases in ISL1 levels were observed in tumor tissues compared with normal adjacent tissues. (B) The protein levels of ISL1 in tumor tissues and normal adjacent tissues were assessed by immunohistochemistry. An increase in ISL1 levels was identified in tumor tissues compared with normal adjacent tissues (magnification, $\mathrm{x} 400$ ). ${ }^{* * *} \mathrm{P}<0.0001$. ISL1, Islet 1 .

significant difference in ISL1 levels was observed for distant metastasis between M0 and M1 (3.13 \pm 1.25 vs. 4.22 \pm 1.49 ; $\mathrm{P}=0.04)$.

ISL1 promotes cell proliferation in TNBC cells. To additionally explore the function of ISL1 in TNBC cells, the present study determined the proliferation of MDA-MB-231 and MDA-MB-468 cells in response to silencing and overexpressing of ISL1. Western blotting confirmed that the ISL1 protein levels in MDA-MB-231 cells were decreased upon silencing of ISL1 compared with those in the control siRNA group. By contrast, the ISL1 protein levels were increased following overexpression of ISL1 in MDA-MB-231 cells compared with those in the pcDNA3 group (Fig. 2A and B).
In addition, silencing ISL1 inhibited the proliferation of MDA-MB-231 and MDA-MB-468 cells compared with that observed in the control siRNA groups, while overexpression of ISL1 promoted cell proliferation in MDA-MB-231 and MDA-MB-468 cells in comparison with cells transfected with pcDNA3 (Fig. 2C-F).

ISL1 improves the invasive ability of TNBC cells. To explore the role of ISL1 in cell invasion, a Transwell assay was used to evaluate the invasion ability of MDA-MB-231 cells transfected with ISL1 siRNA or control siRNA. Silencing ISL1 markedly inhibited the invasion ability of MDA-MB-231 cells compared with that of the control siRNA group cells (Fig. 3A). Conversely, overexpression of ISL1 increased the 
A

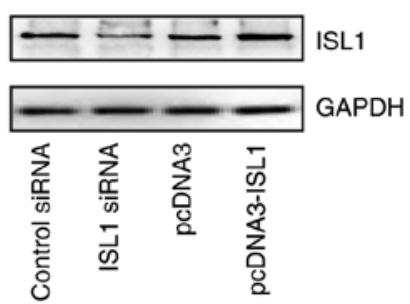

B

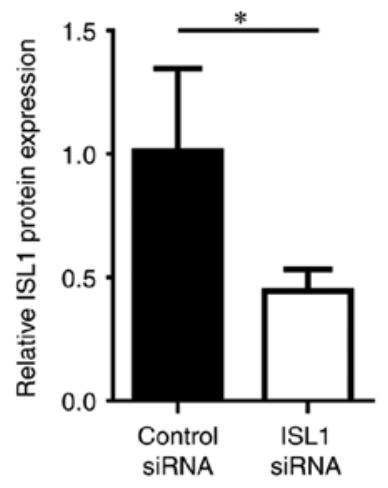

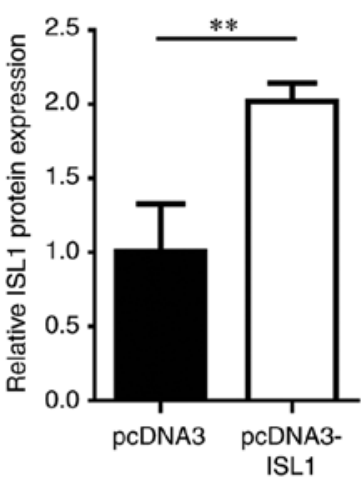

C

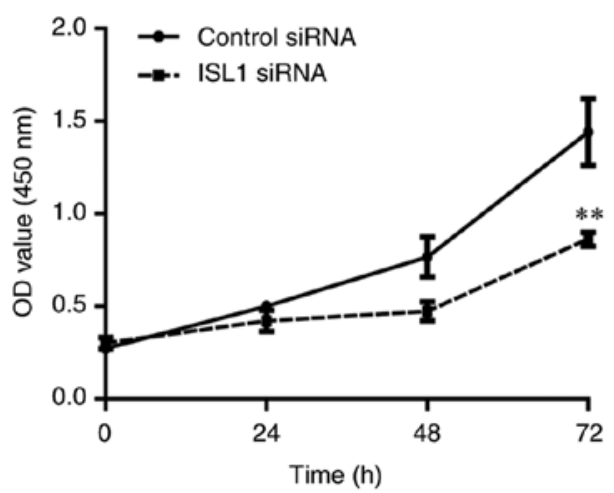

E

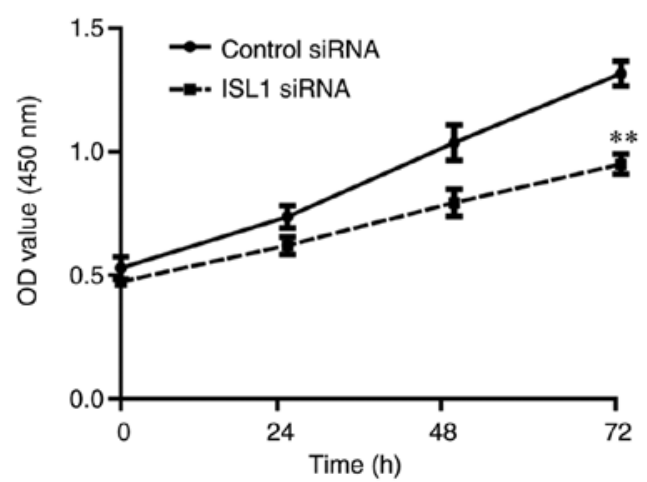

D

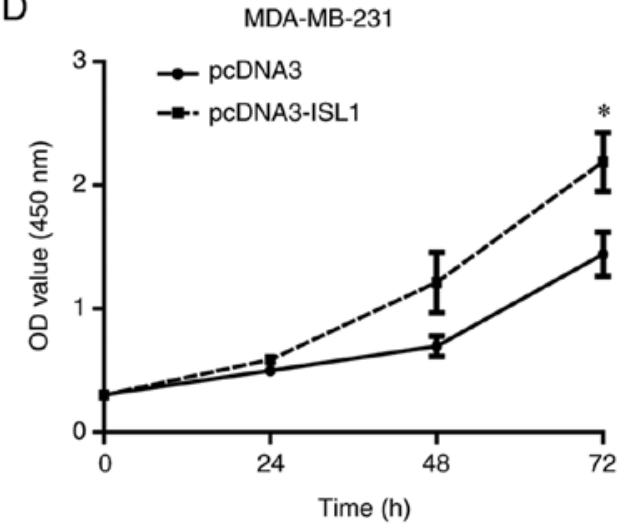

F

MDA-MB-468

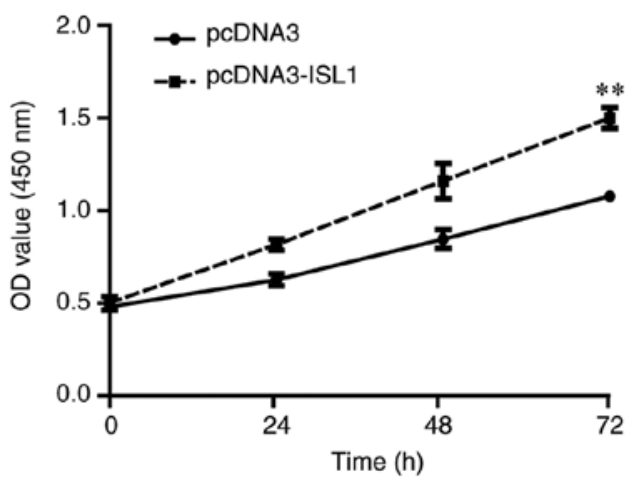

Figure 2. ISL1 promotes cell growth of triple-negative breast cancer cells. (A) In MDA-MB-231 cells, ISL1 siRNA significantly decreased ISL1 protein level compared with control siRNA, and pcDNA3-ISL1 markedly increased ISL1 protein level compared to pcDNA3. (B) Densitometric analysis of data from (A). ${ }^{*} \mathrm{P}<0.05$ vs. control siRNA and ${ }^{* *} \mathrm{P}<0.01$ vs. pcDNA3. (C) ISL1 siRNA markedly inhibited cell proliferation of MDA-MB-231 cells compared with control siRNA. ${ }^{* *} \mathrm{P}<0.01$ vs. control siRNA. (D) pcDNA3-ISL1 significantly accelerated cell growth of MDA-MB-231 cells compared with pcDNA3 ${ }^{*} \mathrm{P}<0.05$ vs. pcDNA3. (E) ISL1 siRNA markedly inhibited cell proliferation of MDA-MB-468 cells compared with control siRNA. ${ }^{* *} \mathrm{P}<0.01$ vs. control siRNA. (F) pcDNA3-ISL1 significantly accelerated cell growth of MDA-MB-468 cells compared with pcDNA3-transfected cells. ${ }^{* *}$ P $<0.01$ vs. pcDNA3. siRNA, small interfering RNA; ISL1, Islet 1.

number of cells that invaded into the lower chamber compared with that observed in the group transfected with pcDNA3 (Fig. 3B). Similar results were observed in MDA-MB-468 cells (Fig. 3C and D). These data indicated that ISL1 may promote the metastasis of TNBC cells.

ISL1 exhibits anti-apoptotic ability in TNBC cells. Next, the present study sought to examine the function of ISL1 in TNBC cell apoptosis. Silencing ISL1 induced a significant but relatively small increase in cell apoptosis in MDA-MB-231 and MDA-MB-468 cells compared with that observed in the control siRNA group (Fig. 4), suggesting that ISL1 is involved in the regulation of cell apoptosis in TNBC cells.
ISL1 inhibits cisplatin-induced cell apoptosis in TNBC cells. As a modest anti-apoptotic ability was observed for ISL1 in MDA-MB-231 cells, it was hypothesized that ISL1 may antagonize the cell apoptosis induced by chemotherapy agents and contribute to chemoresistance. The results revealed that cell proliferation was markedly inhibited following cisplatin treatment in the MDA-MB-231 and MDA-MB-468 cell lines, compared with that observed in the vehicle group. Additionally, cisplatin-induced cell proliferation was significantly decreased by overexpression of ISL1 and enhanced by ISL1 silencing (Fig. 5A and B). Flow cytometry analysis indicated that cisplatin significantly increased apoptosis in TNBC cells compared with that observed in control cells treated with vehicle. 
A

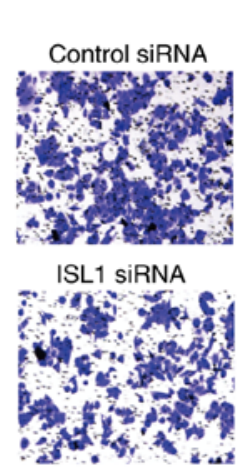

MDA-MB-231

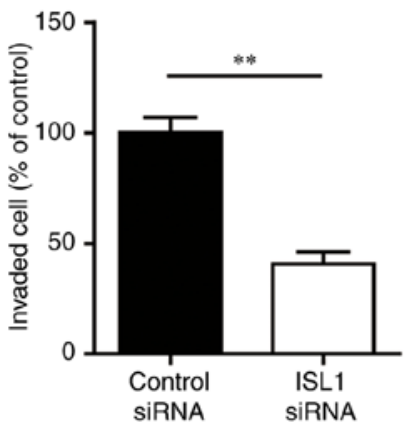

B

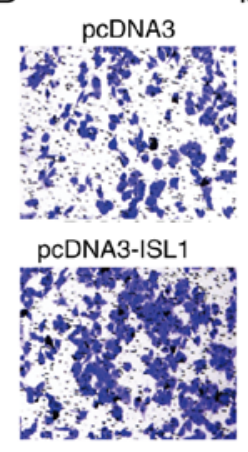

MDA-MB-231

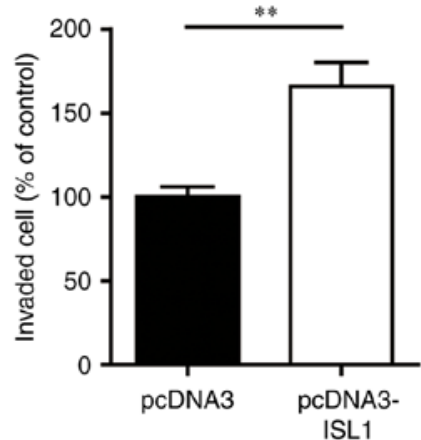

C

MDA-MB-468

D

MDA-MB-468

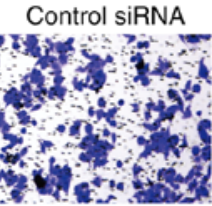

ISL1 SiRNA
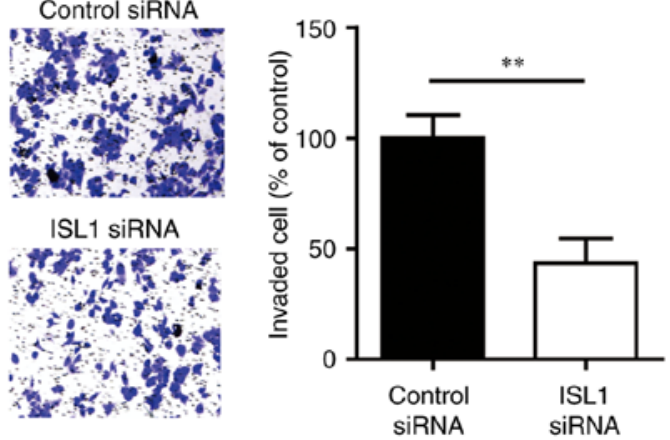

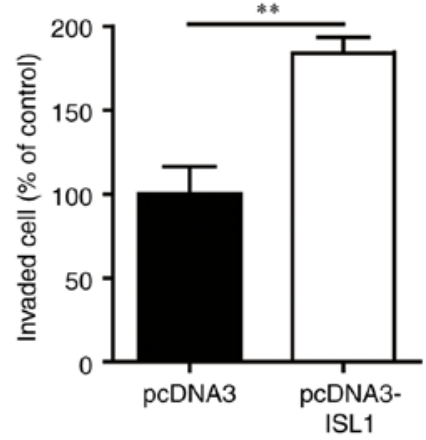

Figure 3. ISL1 contributes to cell invasion of triple-negative breast cancer cells. (A) Compared with cells transfected with control siRNA, the number of invading cells was decreased in MDA-MB-231 cells transfected with ISL1 siRNA. (B) Compared with the cells transfected with pcDNA3, the number of invading cells was increased in MDA-MB-231 cells transfected with pcDNA3-ISL1. (C) Compared with cells transfected with control siRNA, the number of invading cells was decreased in MDA-MB-468 cells transfected with ISL1 siRNA. (D) Compared with cells transfected with pcDNA3, the number of invading cells was increased in MDA-MB-468 cells transfected with pcDNA3-ISL1. ${ }^{* *} \mathrm{P}<0.01$. siRNA, small interfering RNA; ISL1, Islet 1.

A

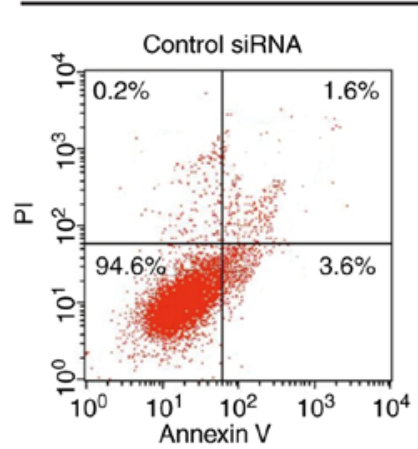

MDA-MB-231

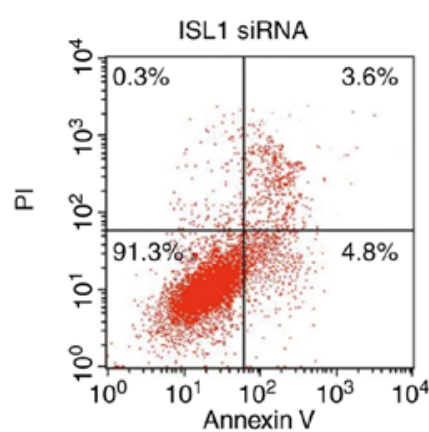

MDA-MB-468

B

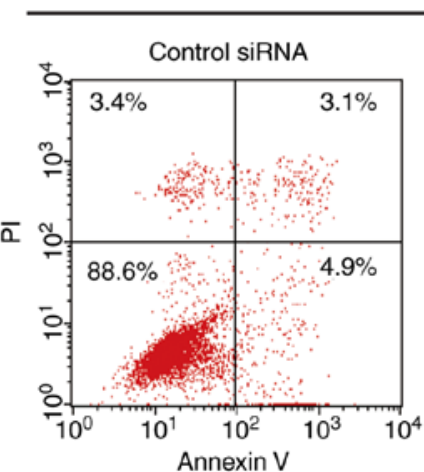

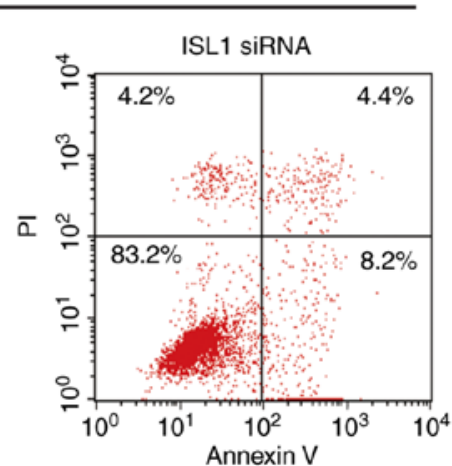
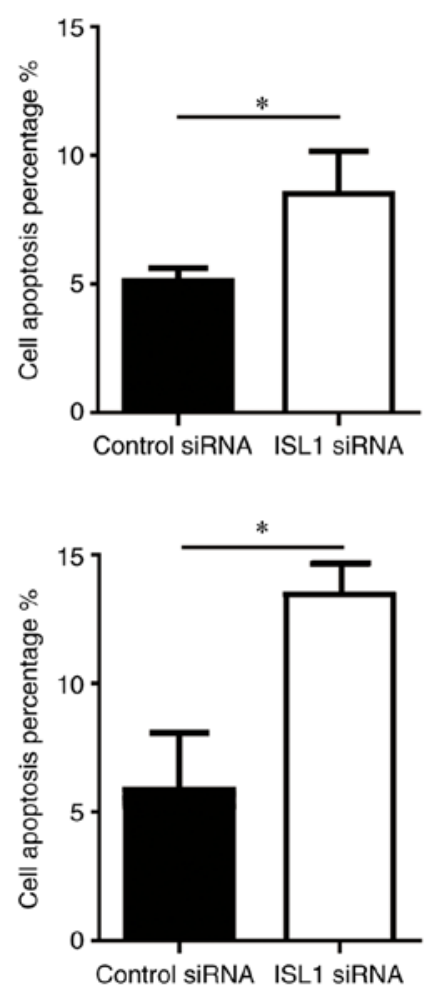

Figure 4. ISL1 exhibits anti-apoptotic ability in triple-negative breast cancer cells. (A) Compared with the control siRNA group, ISL1 siRNA induced a small increase in cell apoptosis of MDA-MB-231. (B) Compared with the control siRNA group, ISL1 siRNA induced a small increase in the cell apoptosis percentage of MDA-MB-468 cells. ${ }^{\text {* }} \mathrm{P}<0.05$. siRNA, small interfering RNA; ISL1, Islet 1. 

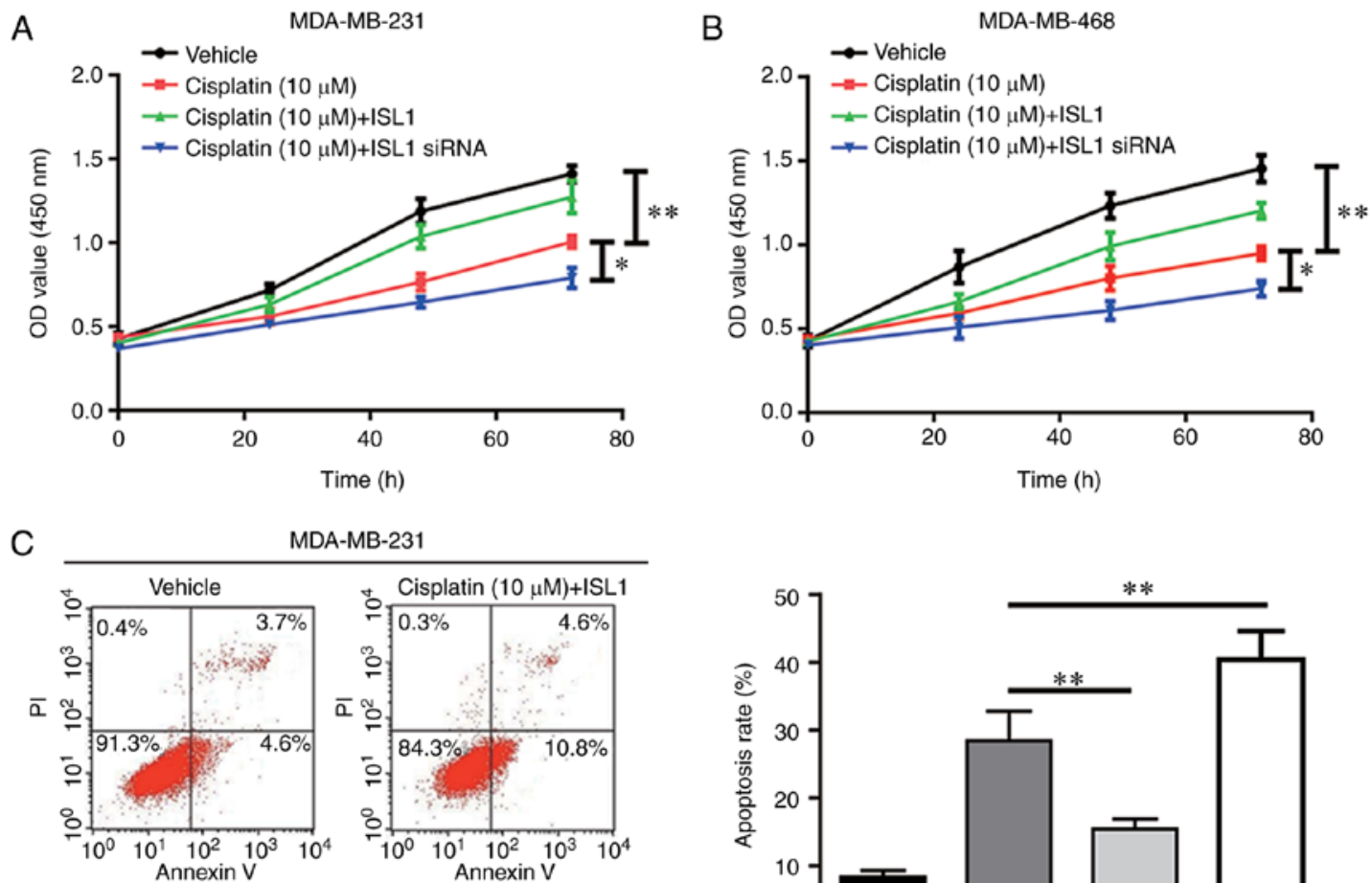

MDA-MB-231
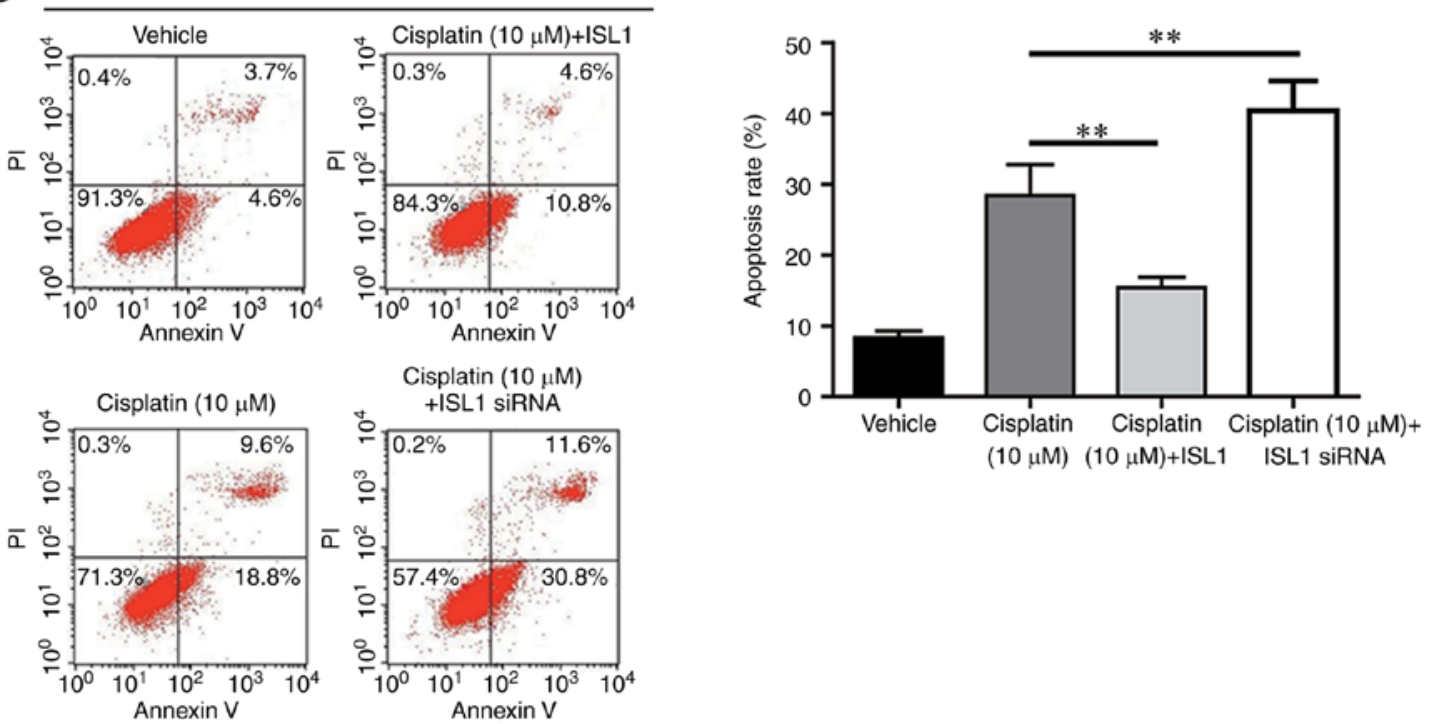

D

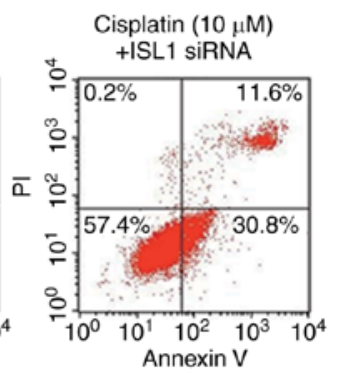

MDA-MB-468
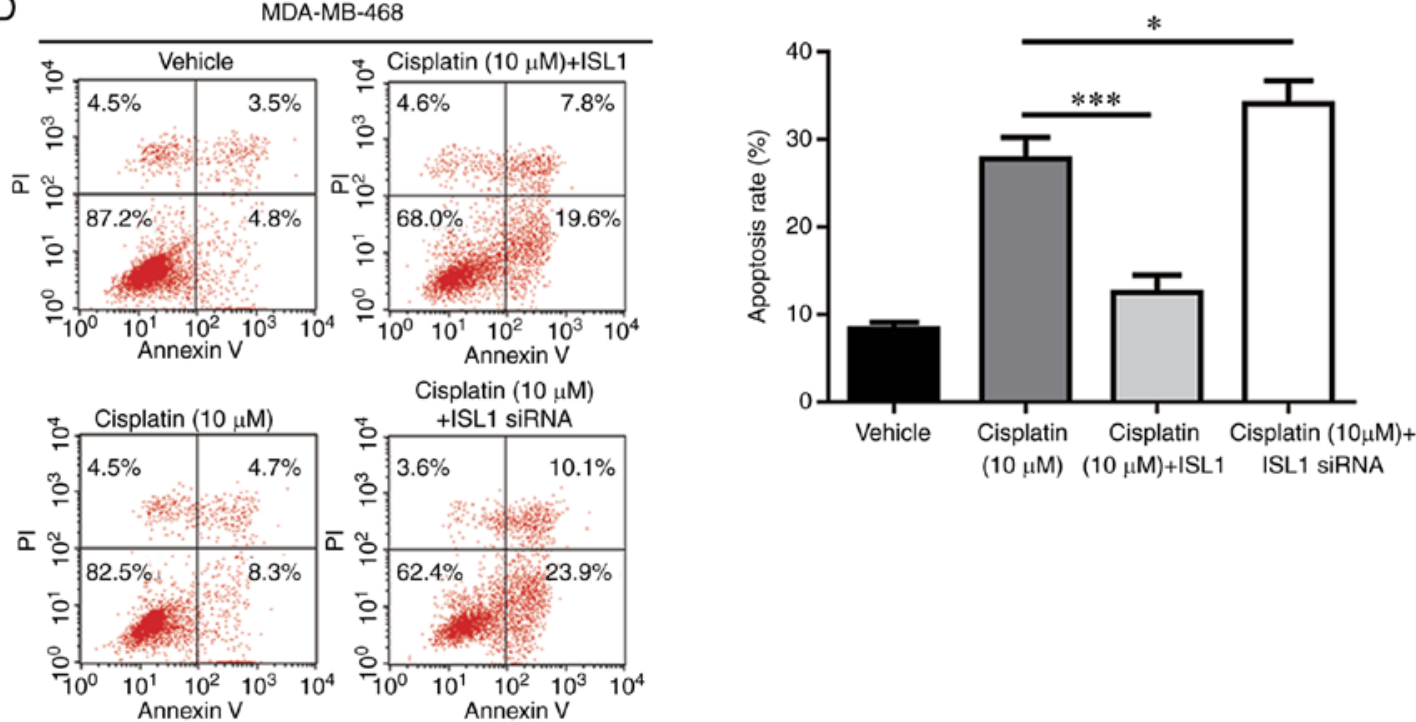

Figure 5. ISL1 decreases cisplatin sensitivity of TNBC cells. (A) Compared with the vehicle group, treatment with $10 \mu \mathrm{M}$ cisplatin induced a significant cell growth arrest, which was partially reversed by ISL1 overexpression and augmented by ISL1 silencing in MDA-MB-231 cells. (B) Compared with the vehicle group, treatment with $10 \mu \mathrm{M}$ cisplatin induced a significant cell growth arrest, which was partially reversed by ISL1 overexpression and augmented by ISL1 silencing in MDA-MB-468 cells. (C) Compared with the vehicle group, significant cell apoptosis was induced by $10 \mu \mathrm{M}$ cisplatin treatment, which was reversed by ISL1 overexpression and augmented by ISL1 silencing in MDA-MB-231 cells. (D) Compared with the vehicle group, significant cell apoptosis was induced by $10 \mu \mathrm{M}$ cisplatin treatment, which was reversed by ISL1 overexpression and augmented by ISL1 silencing in MDA-MB- 468 cells. ${ }^{*} \mathrm{P}<0.05,{ }^{* * *} \mathrm{P}<0.01$ and ${ }^{* * *} \mathrm{P}<0.0001$. siRNA, small interfering RNA; ISL1, Islet 1; OD, optical density; PI, propidium iodide.

In addition, cisplatin-induced cell apoptosis was reversed by ISL1 overexpression and increased by ISL1 silencing in TNBC cells (Fig. 5C and D). These results demonstrated that ISL1 may decrease the sensitivity of TNBC cells towards cisplatin. 
A

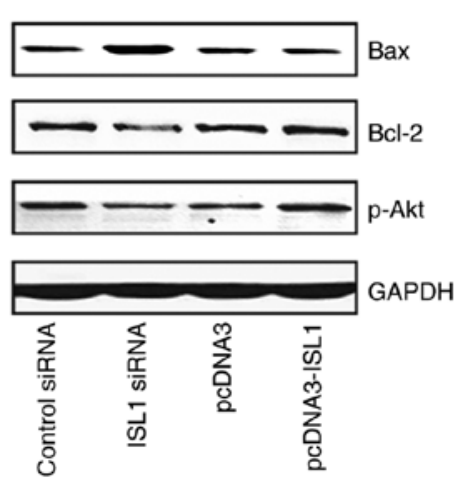

B

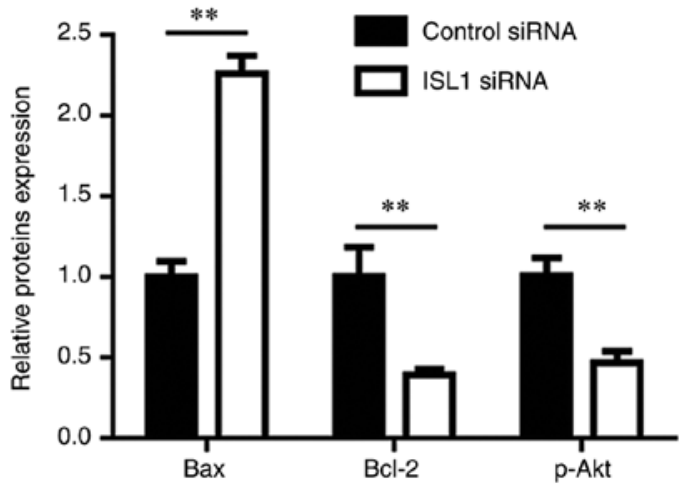

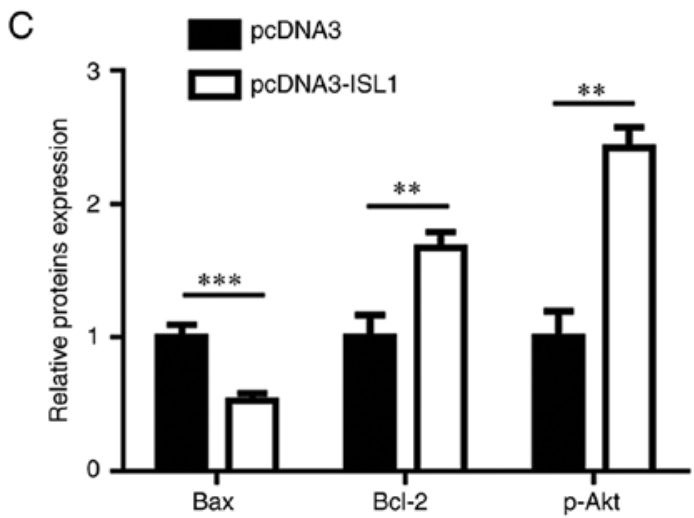

Figure 6. ISL1 regulated expression levels of Bax, Bcl-2 and p-Akt in MDA-MB-231 cells. (A) Western blot analysis indicated that ISL1 siRNA increased Bax protein level, and decreased Bcl-2 and p-Akt protein levels in comparison with those in the control siRNA group in MDA-MB-231 cells. Conversely, pcDNA3-ISL1 decreased Bax protein level, and increased Bcl-2 and p-Akt protein levels when compared with those of the pcDNA3 group. (B) Densitometric analysis of the results from (A). (C) Quantitative analysis of Bax, Bcl-2 and p-Akt protein level in A following overexpression of ISL1, ${ }^{* * *} \mathrm{P}<0.01$ and ${ }^{* * *} \mathrm{P}<0.0001$. Bcl-2, B-cell lymphoma 2; Bax, Bcl-2-associated X protein; Akt, protein kinase B; p-Akt, phosphorylated Akt; siRNA, small interfering RNA; ISL1, Islet 1.

ISL1 regulates pro-apoptotic and anti-apoptotic proteins. To explore the potential molecular mechanism of ISL1 in an anti-apoptosis pathway, the protein levels of pro-apoptotic (Bax) and anti-apoptotic (Bcl-2 and p-Akt) proteins were detected in MDA-MB-231 cells. Silencing ISL1 increased Bax protein levels, and decreased Bcl-2 and p-Akt protein levels (Fig. 6A and B), while overexpression of ISL1 decreased Bax protein levels, and increased Bcl-2 and p-Akt protein levels (Fig. 6A and C). These data indicated that ISL1 is involved in the modulation of cell apoptosis through the regulation of anti-apoptotic and pro-apoptotic proteins.

\section{Discussion}

Breast cancer remains a leading cause of mortality in women worldwide (1). Compared with other types of breast cancer, TNBC is characterized by increased aggressive behavior and relatively poorer clinical outcome (20). The present study revealed that ISL1 was involved in the development of TNBC via regulating cell proliferation, cell invasion and chemotherapy sensitivity in TNBC cells.

Using Affymetrix, ISL1 was first observed to be upregulated in biliary tract cancer tissues compared with normal biliary epithelial scrapings (21). Subsequently, ISL1 was identified as a marker for pancreatic endocrine tumors and metastasis, and was also observed to be overexpressed in rhabdomyosarcoma $(22,23)$. Furthermore, the results of immunohistochemistry in 348 breast cancer tissues indicated that ISL1 expression was notably increased in TNBC tissues compared with that in tissues of other breast cancer sub-types (17). A recent study revealed that ISL1 promoted gastric cancer cell proliferation via the regulation of cyclin-B1, cyclin-B2 and c-Myc (24). The present study revealed that ISL1-knockdown inhibited cell proliferation, while ISL1 overexpression promoted cell proliferation in MDA-MB-231 and MDA-MB-468 cells. In addition, ISL1-knockdown decreased the number of MDA-MB-231 and MDA-MB-468 cells that invaded through Transwell chambers, while ISL1 overexpression increased the invasion ability of these cells. Together, these data suggest that ISL1 promotes the progression of TNBC.

With no targeted therapy, the treatment of patients with TNBC primarily relies on chemotherapy. However, de novo and acquired chemoresistance usually result in poor drug response and eventually lead to patient mortality (25). Numerous genes have been demonstrated to contribute to chemotherapy resistance in $\operatorname{TNBC}(26,27)$. The present study identified that ISL1 knockdown induced a slight increase in cell apoptosis. However, in the presence of cisplatin, ISL1 downregulation notably increased the apoptosis of MDA-MB-231 and MDA-MB-468 cells. Furthermore, ISL1 overexpression was able to reverse cisplatin-induced apoptosis in MDA-MB-231 and MDA-MB-468 cells. The ratio of $\mathrm{Bcl}-2 / \mathrm{Bax}$, which is deregulated in cisplatin-induced cell apoptosis, has been demonstrated to be critical for cell survival $(28,29)$. In addition, previous studies have indicated that high expression levels of p-Akt contribute to cisplatin 
resistance via inhibition of cell apoptosis in various types of cancer $(30,31)$. In the present study, ISL1 silencing markedly increased Bax protein levels, and decreased Bcl-2 and p-Akt protein levels in MDA-MB-231 cells. Conversely, the overexpression of ISL1 markedly decreased Bax levels, and increased Bcl-2 and p-Akt levels. Collectively, the results of the present study indicated that ISL1 may suppress cell apoptosis, particularly cisplatin-induced cell apoptosis, in TNBC cells.

In conclusion, the present study identified ISL1 as a potential oncogene in TNBC, as ISL1 promoted cell proliferation and invasion and inhibited cell apoptosis in TNBC cells. Furthermore, ISL1 overexpression reversed cisplatin-induced cell apoptosis, indicating that ISL1 inhibited the sensitivity of TNBC cells towards cisplatin. Therefore, ISL1 may be a promising therapeutic target and a predictor of cisplatin sensitivity for patients with TNBC.

\section{Acknowledgements}

Not applicable.

\section{Funding}

No funding was received.

\section{Availability of data and materials}

The datasets used and/or analyzed during the current study are available from the corresponding author on reasonable request.

\section{Authors' contributions}

Concept and design: HC. Acquisition of data: YZ, LW, PG, ZS, NL, YL, JSh, JS, HD and YY. Analysis and interpretation of data: HC, YZ, LW, PG and ZS. Writing and review of manuscript: HC, YZ. Study supervision: HC

\section{Ethics approval and consent to participate}

Written informed consent for use of patient samples was obtained from all participants prior to surgery. The experiments were performed following approval from the Ethics Committee of Tangshan People's Hospital.

\section{Patient consent for publication}

Written informed consent for use of patient samples was obtained from all participants prior to surgery.

\section{Competing interests}

The authors declare that they have no competing interests.

\section{References}

1. Patnaik JL, Byers T, DiGuiseppi C, Dabelea D and Denberg TD: Cardiovascular disease competes with breast cancer as the leading cause of death for older females diagnosed with breast cancer: A retrospective cohort study. Breast Cancer Res 13: R64, 2011.

2. Cancer Genome Atlas Network: Comprehensive molecular portraits of human breast tumours. Nature 490: 61-70, 2012.
3. Cheang MC, Voduc D, Bajdik C, Leung S, McKinney S, Chia SK, Perou CM and Nielsen TO: Basal-like breast cancer defined by five biomarkers has superior prognostic value than triple-negative phenotype. Clin Cancer Res 14: 1368-1376, 2008.

4. Oualla K, El-Zawahry HM, Arun B, Reuben JM, Woodward WA, Gamal El-Din H, Lim B, Mellas N, Ueno NT and Fouad TM: Novel therapeutic strategies in the treatment of triple-negative breast cancer. Ther Adv Med Oncol 9: 493-511, 2017.

5. Brasó-Maristany F, Filosto S, Catchpole S, Marlow R, Quist J, Francesch-Domenech E, Plumb DA, Zakka L, Gazinska P, Liccardi G, et al: PIM1 kinase regulates cell death, tumor growth and chemotherapy response in triple-negative breast cancer. Nat Med 22: 1303-1313, 2016.

6. Lehmann BD, Bauer JA, Chen X, Sanders ME, Chakravarthy AB, Shyr Y and Pietenpol JA: Identification of human triple-negative breast cancer subtypes and preclinical models for selection of targeted therapies. J Clin Invest 121: 2750-2767, 2011.

7. Rakha EA, Reis-Filho JS and Ellis IO: Basal-like breast cancer: A critical review. J Clin Oncol 26: 2568-2581, 2008.

8. Hu XC, Zhang J, Xu BH, Cai L, Ragaz J, Wang ZH, Wang BY, Teng YE, Tong ZS, Pan YY, et al: Cisplatin plus gemcitabine versus paclitaxel plus gemcitabine as first-line therapy for metastatic triple-negative breast cancer (CBCSG006): A randomised, open-label, multicentre, phase 3 trial. Lancet Oncol 16: 436-446, 2015.

9. Pruefer FG, Lizarraga F, Maldonado V and Melendez-Zajgla J: Participation of Omi Htra2 serine-protease activity in the apoptosis induced by cisplatin on SW480 colon cancer cells. J Chemother 20: 348-354, 2008.

10. Rottenberg S, Nygren AO, Pajic M, van Leeuwen FW, van der Heijden I, van de Wetering K, Liu X, de Visser KE, Gilhuijs KG, van Tellingen $\mathrm{O}$, et al: Selective induction of chemotherapy resistance of mammary tumors in a conditional mouse model for hereditary breast cancer. Proc Natl Acad Sci USA 104: 12117-12122, 2007.

11. Galluzzi L, Senovilla L, Vitale I, Michels J, Martins I, Kepp O, Castedo $\mathrm{M}$ and Kroemer G: Molecular mechanisms of cisplatin resistance. Oncogene 31: 1869-1883, 2012.

12. Brozovic A and Osmak M: Activation of mitogen-activated protein kinases by cisplatin and their role in cisplatin-resistance. Cancer Lett 251: 1-16, 2007.

13. Zhou BP, Liao Y, Xia W, Spohn B, Lee MH and Hung MC: Cytoplasmic localization of p21Cip1/WAF1 by Akt-induced phosphorylation in HER-2/neu-overexpressing cells. Nat Cell Biol 3: 245-252, 2001

14. Karlsson O, Thor S, Norberg T, Ohlsson H and Edlund T: Insulin gene enhancer binding protein Isl-1 is a member of a novel class of proteins containing both a homeo- and a Cys-His domain. Nature 344: 879-882, 1990.

15. Hobert $\mathrm{O}$ and Westphal H: Functions of LIM-homeobox genes. Trends Genet 16: 75-83, 2000.

16. Guo C, Wang W, Shi Q, Chen P and Zhou C: An abnormally high expression of ISL-1 represents a potential prognostic factor in gastric cancer. Hum Pathol 46: 1282-1289, 2015.

17. Shin E, Lee Y and Koo JS: Differential expression of the epigenetic methylation-related protein DNMT1 by breast cancer molecular subtype and stromal histology. J Transl Med 14: 87, 2016.

18. Livak and Schmittgen: Analysis of relative gene expression data using real-time quantitative PCR and the 2(-Delta Delta C(T)) method. Methods 25: 402-408, 2001.

19. Elston CW and Ellis IO: Pathological prognostic factors in breast cancer. The value of histologic grade in breast cancer: Experience from a large study with long term follow-up. Histopathology 19: 403-410, 1991.

20. Kong X, Liu W and Kong Y: Roles and expression profiles of long non-coding RNAs in triple-negative breast cancers. J Cell Mol Med 22: 390-394, 2018.

21. Hansel DE, Rahman A, Hidalgo M, Thuluvath PJ, Lillemoe KD, Schulick R, Ku JL, Park JG, Miyazaki K, Ashfaq R, et al: Identification of novel cellular targets in biliary tract cancers using global gene expression technology. Am J Pathol 163: 217-229, 2003.

22. Schmitt AM, Riniker F, Anlauf M, Schmid S, Soltermann A, Moch H, Heitz PU, Klöppel G, Komminoth P and Perren A: Islet 1 (Is11) expression is a reliable marker for pancreatic endocrine tumors and their metastases. Am J Surg Pathol 32: 420-425, 2008.

23. Erlenbach-Wünsch K, Haller F, Taubert H, Wurl P, Hartmann A and Agaimy A: Expression of the LIM homeobox domain transcription factor ISL1 (Islet-1) is frequent in rhabdomyosarcoma but very limited in other soft tissue sarcoma types. Pathology 46 : 289-295, 2014 
24. Shi Q, Wang W, Jia Z, Chen P, Ma K and Zhou C: ISL1, a novel regulator of CCNB1, CCNB2 and c-MYC genes, promotes gastric cancer cell proliferation and tumor growth. Oncotarget 7: 36489-36500, 2016.

25. Schlotter CM, Tietze L, Vogt U,Heinsen CV and Hahn A: Ki67 and lymphocytes in the pretherapeutic core biopsy of primary invasive breast cancer: Positive markers of therapy response prediction and superior survival. Horm Mol Biol Clin Investig: Sep 22, 2017 (Epub ahead of print). doi: https://doi.org/10.1515/hmbci-2017-0022.

26. Wein L and Loi S: Mechanisms of resistance of chemotherapy in early-stage triple negative breast cancer (TNBC). Breast 34 (Suppl 1): S27-S30, 2017.

27. Gómez-Miragaya J, Palafox M, Paré L, Yoldi G, Ferrer I, Vila S, Galván P, Pellegrini P, Pérez-Montoyo H, Igea A, et al: Resistance to taxanes in triple-negative breast cancer associates with the dynamics of a CD $49 \mathrm{f}+$ tumor-initiating population. Stem Cell Reports 8: 1392-1407, 2017.

28. Saxena A, Viswanathan S, Moshynska O, Tandon P, Sankaran K and Sheridan DP: $\mathrm{Mcl}-1$ and $\mathrm{Bcl}-2 / \mathrm{Bax}$ ratio are associated with treatment response but not with Rai stage in B-cell chronic lymphocytic leukemia. Am J Hematol 75: 22-33, 2004.
29. Chen L, Cui H, Fang J, Deng H, Kuang P, Guo H, Wang X and Zhao L: Glutamine deprivation plus BPTES alters etoposide- and cisplatin-induced apoptosis in triple negative breast cancer cells. Oncotarget 7: 54691-54701, 2016.

30. Liu S, Ren B, Gao H, Liao S, Zhai YX, Li S, Su XJ, Jin P, Stroncek D, Xu Z, et al: Over-expression of BAG-1 in head and neck squamous cell carcinomas (HNSCC) is associated with cisplatin-resistance. J Transl Med 15: 189, 2017.

31. Wen Q, Liu Y, Lyu H, Xu X, Wu Q, Liu N, Yin Q, Li J and Sheng X: Long noncoding RNA GAS5, which acts as a tumor suppressor via microRNA 21, regulates cisplatin resistance expression in cervical cancer. Int J Gynecol Cancer 27: 1096-1108, 2017.

This work is licensed under a Creative Commons Attribution-NonCommercial-NoDerivatives 4.0 International (CC BY-NC-ND 4.0) License. 